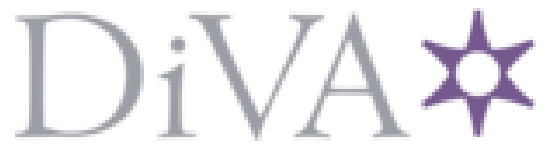

http://www.diva-portal.org

Preprint

This is the submitted version of a paper published in IEEE Sensors Journal.

Citation for the original published paper (version of record):

Ashraf, S., Mattsson, C., Thungström, G. (2019)

Fabrication and characterization of a SU8-epoxy membrane based thermopile detector with an integrated multilayered absorber structure for the mid-IR region

IEEE Sensors Journal

https://doi.org/10.1109/JSEN.2019.2896668

Access to the published version may require subscription.

N.B. When citing this work, cite the original published paper.

Permanent link to this version:

http://urn.kb.se/resolve?urn=urn:nbn:se:miun:diva-35525 
“C 20XX IEEE. Personal use of this material is permitted. Permission from IEEE must be obtained for all other uses, in any current or future media, including reprinting/republishing this material for advertising or promotional purposes, creating new collective works, for resale or redistribution to servers or lists, or reuse of any copyrighted component of this work in other works." 


\title{
Fabrication and characterization of a SU8-epoxy membrane based thermopile detector with an integrated multilayered absorber structure for the mid-IR region
}

\author{
Shakeel Asharf, Claes G. Mattsson, Göran Thungström
}

\begin{abstract}
This paper reports on the fabrication and characterization of a thermopile detector with an integrated midinfrared absorber structure. The fabricated absorber structure has shown an absorption of more than $95 \%$ in the wavelength range of $3.2-5.47 \mu \mathrm{m}$. The detector was fabricated with standard cleanroom process techniques and equipment. The serial resistance was measured at about $315 \mathrm{k} \Omega$ at room temperature. The photosensitivity of the detector was characterized for a signle wavelength $(4.26 \mu \mathrm{m})$ and a band of wavelength ranging from $2.5-$ $5.5 \mu \mathrm{m}$ through two different measurement setups. In the first measurement setup, the photosensitivity was estimated at $57.5 \mathrm{~V} \cdot \mathrm{mm}^{2} \cdot \mathrm{W}^{-1}$ through a MEMS-based infrared radiation source and with an optical band-pass filter of wavelength $4.26 \mu \mathrm{m}$. The following characterization was performed to characterise the photosensitivity of the detector in a broader wavelength range. This measurement was taken using a monochromator setup utilizing a reference photodetector for calculations of the optical power of the infrared source. The photosensitivity and the specific detectivity $\left(D^{*}\right)$ of the fabricated detector were measured to values of $30-92 \mathrm{~V} \cdot \mathrm{W}^{-1}$ and $8.0 \times 10^{7}-2.4 \times 10^{8} \mathrm{~cm} \cdot \mathrm{Hz}^{1 / 2} \cdot \mathrm{W}^{-1}$, respectively, in the wavelength range of $2.8-5 \mu \mathrm{m}$. The time constant was estimated to around $21 \mathrm{~ms}$.
\end{abstract}

Keywords-Interferometric absorber; thermopile detector; photosensitivity; SU-8 epoxy ; Transfer matrix theory.

\section{INTRODUCTIONS}

$\mathrm{T}$ HERMAL detectors play an important role in different types of gas detection systems/applications. Room temperature operation of thermal detectors, such as the microthermopiles and bolometers, gives thermal detectors a significant advantage over photon detectors. The cooling requirement of photon detectors makes them bulky and expensive and, therefore, many cost-sensitive applications utilize thermal detectors. The use of this type of detector can be found in various applications, and an overall review of infrared (IR) detectors is presented in [1].

\footnotetext{
${ }^{\mathrm{T}}$ he revised manuscript was submitted on 04 of March 2018. This paper is an extension of the original paper presented at the IEEE Sensor Conference 2016, which was published in its proceedings. The link to the original paper is provided here:

http://ieeexplore.ieee.org/document/7808481/ DOI: 10.1109/ICSENS.2016.7808481
}

The sensitivity/performance of thermal detectors could effectively be increased by selecting membrane materials with low thermal conductivity values and an efficient infrared (IR) absorbing material/structure. Materials such as SU-8 epoxy have a very low thermal conductivity value of $0.2-0.3 \mathrm{~W} / \mathrm{mK}[2][3]$. This should be compared to other commonly used membrane materials, such as silicon and silicon nitrate, with thermal conductivity values of $150 \mathrm{~W} / \mathrm{mK}$ [4] and $2.3-30 \mathrm{~W} / \mathrm{mK}[5]$ respectively. Additionally, these membrane materials require advanced and expensive equipment, i.e. low pressure chemical vapour deposition or plasma-enhanced chemical vapour deposition equipment. The chemical vapour deposition process involves handling hazardous gases, which should be handled with care. In relation to this, the SU-8 deposition process only includes standard photo-lithographic processing steps, such as spin-coating and UV lithography. Such equipment is commonly available in most of the process facilities for detector fabrication labs. Furthermore, SU-8 is chemically resistant to most solvents and acids, and it is thermally stable up to $300{ }^{\circ} \mathrm{C}$. The fabrication of an $\mathrm{Al} / \mathrm{Bi}$ thermopile detector using SU-8 epoxy as a membrane material has been reported in [6].

Graphite-based black paint has absorption values from 80 to $93 \%$ for a wide spectral range of wavelength $2.5-20 \mu \mathrm{m}$. The deposition of this type of absorber has been performed either by spraying directly onto the detector surface as reported in [7] or by using a very small brush. The use of a black paint as an IR-absorber has been reported in [8]. The deposition of such a layer is difficult in cleanroom facilities. These deposition methods could result in contamination, which restricts their use in cleanroom facilities. The membranes of the thermopile detector are very thin and fragile, and a rough deposition method could damage them. In addition, a thick and an uneven distribution of the absorber layer over the hot junctions would eventually increases the thermal capacitance of the detector, which results in a longer thermal response time.

This work was carried out at the Research Centre STC (Sensible things that communicate) at Mid Sweden University. STC is sponsored by the Knowledge Foundation in Sweden.

S. Ashraf, C. G. Mattsson, and G. Thungström are with the Department of Electronics Design at Mid Sweden University, SE-85170 Sundsvall, Sweden. (shakeel.ashraf@miun.se). 


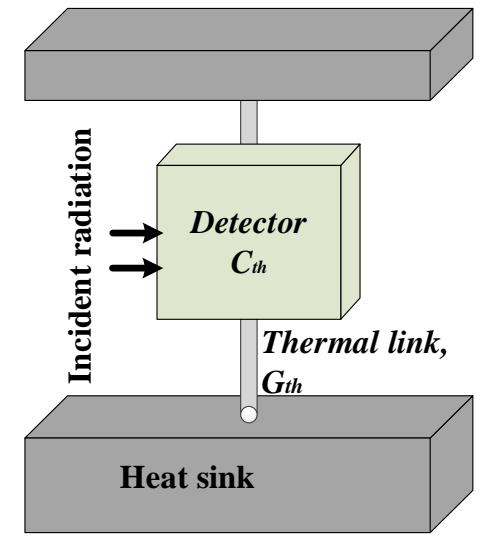

Fig. 1: A simple representation of a thermal detector of thermal conductance Cth, connected with supporting material with a thermal link of thermal capacitance $G_{t h}$.

Thin metal films of sheet resistance $188 \Omega / \square$ could absorb $50 \%$ of incident radiation, as reported in [9]. The combination of a thin metal film (semi-transparent layer) with a dielectric medium of thickness $\lambda / 4$ and a perfect reflector is known as an interferometric $(\lambda / 4)$ absorber. In theory, this structure has an absorption of $100 \%$ at a specific wavelength reported in [10], [11]. The construction of a $\lambda / 4$ IR absorber structure based on SU-8 epoxy (as a dielectric medium) and its integration into the thermopile detector has been reported in [12], where the absorber structure, which was designed for a wavelength of $4.26 \mu \mathrm{m}$, covers $81 \%$ of the detector surface - the surface area of the detector was $4 \mathrm{~mm}^{2}$ andthe absorber had an area of 3.24 $\mathrm{mm}^{2}$. The FTIR measurement showed more than 97\% absorption for the structure. Due to processing limitations and variations, the maximum absorption of the $\lambda / 4$ structure could possibly shift in wavelength as reported in [13], which eventually reduced the detector performance. However, the $\lambda / 4$ structure could be improved by adding additional layers of dielectric medium and semitransparent metal. A multilayered $\lambda / 4$ absorber structure has been reported in [14], where the structure was simulated for range of wavelength 3-6 $\mu \mathrm{m}$, and the FTIR results have shown more than $95 \%$ absorption in the range of $3.2-5.47 \mu \mathrm{m}$. Since the multilayered structure utilizes SU-8 epoxy as a dielectric medium, this allows for a direct integration of this structure into the membrane. The fabrication and the integration of the absorber structure into the membrane of the thermopile has been reported in [15], where the detector voltage response and photosensitivity for a wavelength of $4.26 \mu \mathrm{m}$ was also presented. In this extended paper, the thermopile detector was characterized for photosensitivity $\mathrm{V} \cdot \mathrm{W}^{-1}$, and specific detectivity $\mathrm{D}^{*}$ for the wavelength range of 2.5-5.5 $\mu \mathrm{m}$. The details about the fabrication and characterization are given in the following sections of the article.

\section{Thermal DeteCtors}

A simple representation of a thermal detector is shown in Fig. 1, where a detector of thermal capacitance $\left(\mathrm{C}_{t h}\right)$ is connected to a heat sink via a thermal link of thermal conductance $\left(G_{t h}\right)$ at a normal temperature $(T)$. When radiation is incident on the detector, the radiation-sensitive material absorbs the incident radiation, and this results in a change in

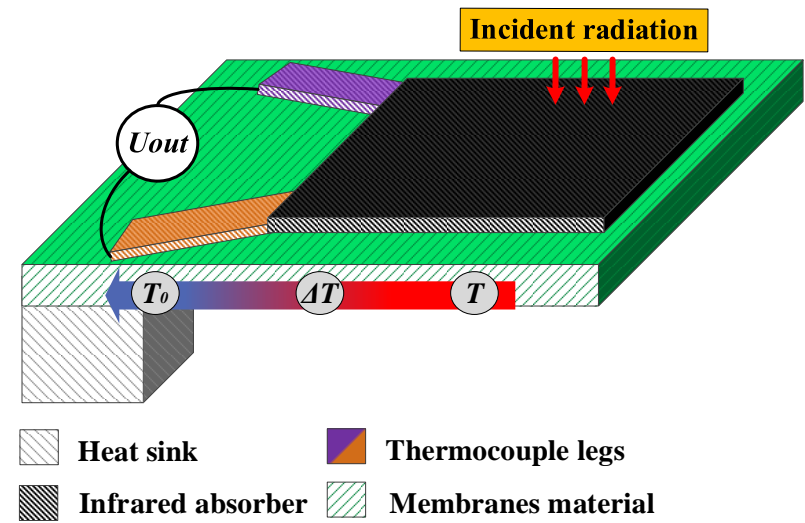

Fig. 2: A simple representation of a thermocouple detector fabricated on selfsupporting membranes, one junction is connected to a heat sink and the other connected to an IR-absorber.

temperature $(\Delta T)$. This change in temperature can be found by solving the heat equation in (1)[16]

$$
C_{t h} \frac{d T}{d t}+G_{t h} \times \Delta T=\varepsilon \times \phi
$$

where, $(\varphi)$ is the incident radiation flux and $(\varepsilon)$ is the emissivity of the active area. If the incident radiation flux is assumed to be periodic, the change in temperature $(\Delta T)$ is given by (2)[17].

$$
\Delta T=\frac{\varepsilon \phi}{\left(G_{t h}^{2}+\omega^{2} C_{t h}^{2}\right)^{1 / 2}}
$$

where, $(\omega)$ is the angular frequency of the incident radiation flux. From (2), we can observe that the change in temperature $(\Delta T)$ could effectively be increased by reducing both the thermal capacitance $\left(C_{t h}\right)$ and thermal conductance $\left(G_{t h}\right)$ values. Whereas to achieve fast detector response given in (3) [17], the value of $\left(C_{t h}\right)$ should be low and the values of $\left(G_{t h}\right)$ should be high. So, there is a trade-off between sensitivity and thermal response.

$$
\tau_{t h}=C_{t h} / G_{t h}
$$

There is one way to increase the overall thermal response and sensitivity: by obtaining thermal isolation between heat sink and radiation sensitive element. Thermal insulation can be obtained by fabricating a self-supporting membrane structure for the thermopile detector. In Fig. 2, a simple representation of a thermopile detector is shown. Here, a cold junction lies on the heat-sink, and the hot junction connected with the IR-absorber and thermocouple is supported by the membrane material. When the IR-absorber receives incident radiation, the voltage response generated across the thermopile detector is directly proportional to the temperature difference and the number of thermocouples; this is known as the Seebeck effect, and the mathematical form is given by (4)

$$
\Delta V=S_{A B}(T) \times \Delta T \times N
$$


where, $S_{A B}=\left|S_{A}-S_{B}\right|, S_{A}$ and $S_{B}$ are the Seebeck coefficients of the materials, $\Delta T=\left|T_{2}-T_{1}\right|, T_{2}$ and $T_{1}$ are the temperature of the hot and cold junctions, and $N$ is the number of thermocouples. The Seebeck coefficient for $\mathrm{Al}$ metal is equal to $+4 \mu \mathrm{V} \cdot \mathrm{K}^{-1}$ and for $\mathrm{Bi}$ it is equal to $-70 \mu \mathrm{V} \cdot \mathrm{K}^{-1}$.

\section{MULTILAYERED STRUCTURE}

\section{A. Simulation of the absorber structure}

Absorption in the structure was calculated by using (5), where, $T_{o p}=$ transmittance, $A_{o p}=$ absorption, $R_{o p}=$ reflectance, and reflectance and transmittance coefficients were calculated by using the transfer matrix theory of multilayer structures [12]. The transfer matrix for each layer in the structure is described by (6).

$$
\begin{gathered}
A_{o p}=1-R_{o p}-T_{o p} \\
{\left[\begin{array}{cc}
\cos (\varphi) & \frac{-i}{n_{l}} \sin (\varphi) \\
-i n_{l} \sin (\varphi) & \cos (\varphi)
\end{array}\right]}
\end{gathered}
$$

In (6) $\varphi=2 \pi t n_{l} / \lambda_{0}$ and $n_{l}$ is the complex reflective indices of the layer, ' $t$ ' is the thickness of the layer and ' $\lambda_{0}{ }^{\prime}$ ' is the optical wavelength. The overall transfer matrix is obtained by multiplying the individual matrices together such as

$$
M_{1} \times M_{2} \times M_{3} \ldots M_{j}=\left[\begin{array}{cc}
A & B \\
C & D
\end{array}\right]
$$

The reflectance $R_{o p}$ and transmittance $T_{o p}$ are calculated by (8), and (9)

$$
T_{o p}=\operatorname{Re}\left(n_{T}\right) \times|t|^{2} / n_{0} \quad, \quad R_{o p}=\operatorname{Re}\left(n_{T}\right) \times|r|^{2} / n_{0}
$$

Where ' $r$ ', and ' $\mathrm{t}$ ' are the reflectance and transmission coefficients given in (8), and (9).

$$
\begin{aligned}
& r=\frac{A \times n_{0}+B \times n_{0} \times n_{T}-C-D \times n_{T}}{A \times n_{0}+B \times n_{0} \times n_{T}+C+D \times n_{T}} \\
& t=\frac{2 \times \mathrm{n}_{T}}{A \times n_{0}+B \times n_{0} \times n_{T}+C+D \times n_{T}}
\end{aligned}
$$

Where $n_{0}$ and $n_{T}$ are the refractive index values of the infinite media above and below the structure, if the air is on both sides, then $n_{0}$ and $n_{T}$ will become ' 1 '. The simulation of the structure presented has been performed in MATLAB by implementing the transfer matrix theory. In the simulation, the thickness of the layers is selected to obtain maximum absorption in the wavelength region of $3 \mu \mathrm{m}-6 \mu \mathrm{m}$. In simulation, refractive index values of the metals were taken from [18]. The index values of SU-8 epoxy are only known for the UV-visible region. In our simulation, we have considered

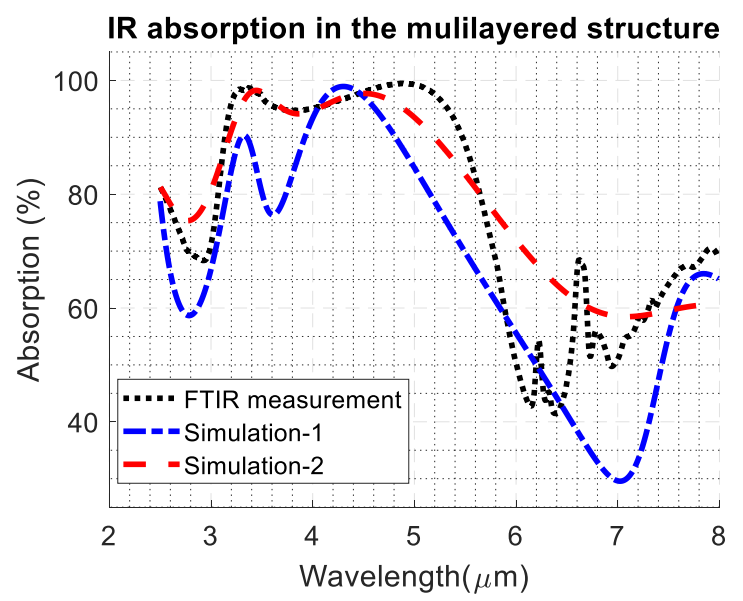

Fig. 3: Black dotted line represents FTIR measurements, blue dash-dot line represents the simulation results without considering the absorption in SU8, and red dashed line represents the simulation results with considering absorption in SU8.

$n_{0}=1.571$, and $k=0$. The simulation result for the multilayered structure is shown in Fig. 3 (Simulation-1).

\section{B. Fabrication and FTIR results}

A multilayered absorber structure was fabricated. This structure consists of a stack of five layers. The bottom layer is

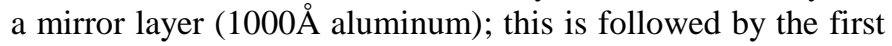
dielectric medium layer of SU-8 epoxy $(2 \mu \mathrm{m})$, then by a titanium metal layer $(200 \AA)$, a dielectric medium layer of SU-8 $(0.6 \mu \mathrm{m})$, and finally by a second titanium metal layer $(75 \AA)$, and the cross-sectional view is shown in Fig. 4f. The Fourier transform infrared spectroscopy (FTIR) measurement is shown in Fig. 3 (FTIR measurement). The initial results showed a rather high mismatch between simulation and measurement. The mismatch was investigated, and it was found that SU-8 epoxy has $10-20 \%$ absorption in the IR region $3-6 \mu \mathrm{m}$ reported in [14]. By introducing ' $\mathrm{k}$ ' the absorption values of SU-8 epoxy into the simulation and FTIR measurement showed better correspondence shown in Fig. 3 (Simulation-2). The multilayered absorber structure shows more than 95\% absorption in the wavelength range $3.2-5.47 \mu \mathrm{m}$ in FTIR measurements and $3.30-5.0 \mu \mathrm{m}$ in simulations.

\section{DETECTOR FABRICATION}

\section{A. Detector fabrication}

The detector was fabricated using standard processing techniques. The fabrication process includes oxidation of the silicon wafer and wet and plasma etching of the silicon dioxide and SU-8 epoxy material, respectively. The photolithography processes include etching, lift-off processes and thermal evaporation of different metals. The cross-sectional view of the fabricated thermopile detector is illustrated in Fig. 4, and the overall detector fabrication process is described below.

Silicon wafer of type ' $n$ ', orientation $\langle 100\rangle$, polished on both sides, was selected as the bulk material (substrate). A $5000 \AA$ thick layer of oxide was formed by a thermal oxidation process, as shown in Fig. 4a. Following this, square openings were formed in the oxide layer on the back for the etching of the silicon. Then, thermocouples $(\mathrm{Al} / \mathrm{Bi})$ were formed on the front side of the substrate by using a photolithographic and lift- 

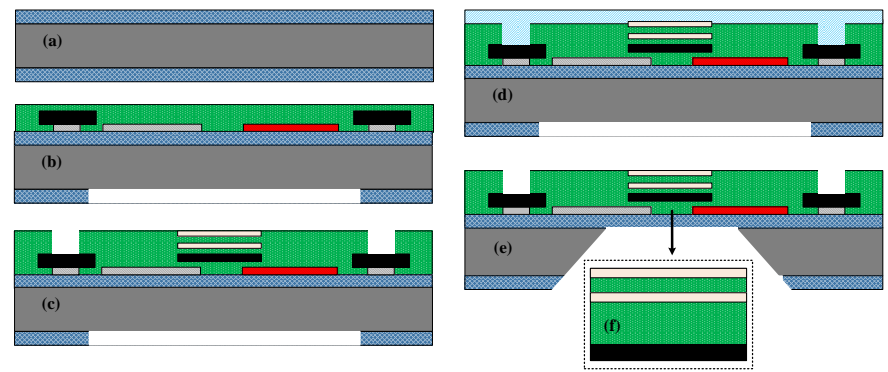

Silicon dioxide

SU-8 epoxy materials

Aluminium

Silicon substrate

Titanium Multilayer Structure

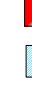

ProTEK B3

Fig. 4: A step-by-step fabrication process of the integration (of an absorber structure) and fabrication of a thermopile detector

off process. A thin layer of titanium was deposited before each metal evaporation in order to promote adhesion between the substrate and thermocouples. For electrical connection, aluminum contact pads were formed in a later process step. Later in the process, a layer of SU-8 epoxy was formed by spin coating process so as to insulate the thermocouples from the multilayer structure. These steps can be seen in Fig. 4b, including openings for contact pads. Metals, such as aluminum, have poor adhesion on SU-8 epoxy, which results in device yield problems.Oxygen gas plasma treatment enhances adhesion of metal on polymers as reported in [19]. Thus, waffer was treated with an RF oxygen plasma to enhance the metal adhesion on SU-8 epoxy.

The fabrication process continued with the integration of the multilayer structure into the membrane of the thermopile detector. Initially, a mirror layer of aluminum with a thickness of $1000 \AA$ was deposited. This was followed by spin coating of a $2 \mu \mathrm{m}$ SU-8 epoxy and evaporation of a $200 \AA$ titanium layer. The fourth layer of the structure was another layer of SU-8 epoxy with a thickness of $0.6 \mu \mathrm{m}$; in the fifth and final stage, a $75 \AA$ titanium layer acting as semitransparent mirror was deposited. The cross-sectional view of the integrated multilayer structure is illustrated in Fig. $4 \mathrm{c}$ and a magnified view of the integrated multilayer structure is shown in Fig. 4f. Following the integration process, Pro-TEK B3 reported in [20] was used as an etch protection on the front of the detector as shown in Fig. 4d. After the completion of the etching process the crosssectional view of the $\mathrm{Al} / \mathrm{Bi}$ - thermopile detector is shown in Fig. 4e. A top view photograph of the thermopile detector is shown

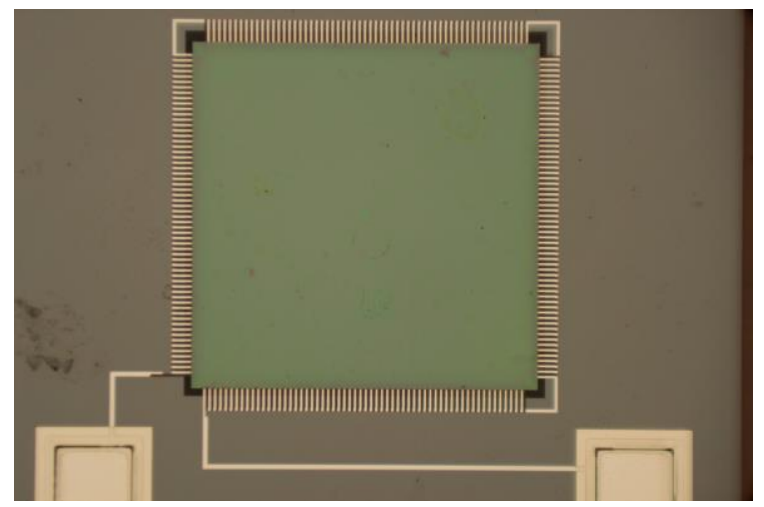

Fig. 5: Top view of the fabricated thermopile detector with integrated multilayered structure.

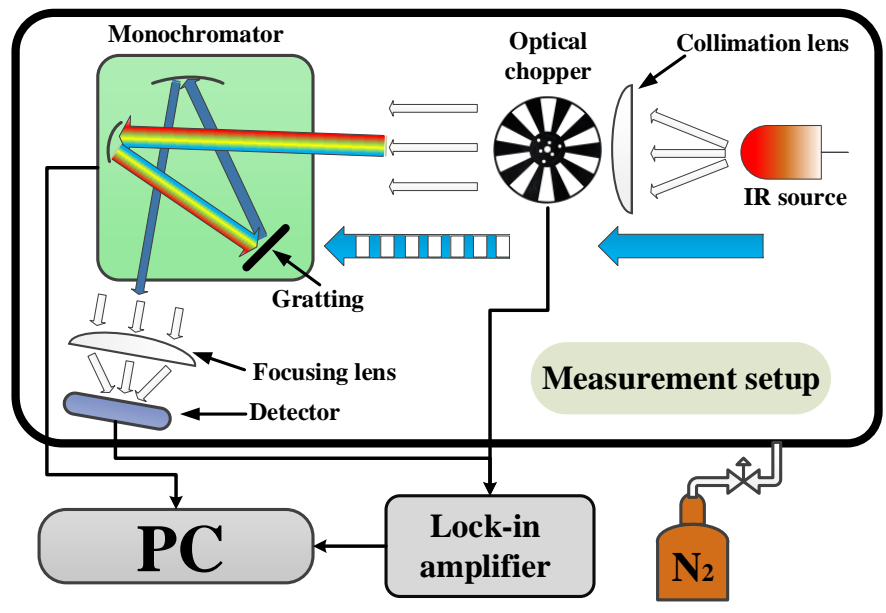

Fig. 6: A block diagram of the measurement setup used for the photosensitivity measurement of the thermopile detector.

in Fig. 5. Here, the green part on the detector is the absorber structure.

\section{DETECTOR MEASUREMENT PREPARATION}

\section{A. Measurement Setup}

A block diagram of the whole measurement setup is shown in Fig. 6. TRIAX 180 monochromator from Horiba Scientific [21] was used in this measurements. A grating for the mid-IR region $2-6 \mu \mathrm{m}$ wavelength was installed in the monochromator. The grating has a groove density of 300 $\mathrm{gr} / \mathrm{mm}$, and the spectral efficiency curve of the grating is available at [22]. IR-18 from SciTec Instruments [23] was used as an IR power source that was positioned at the input of the monochromator. Plano convex lenses were used for the collimation of the input signal and for focusing of the output signal to the detector. An optical chopper was used to modulate the input signal. The infrared source, the convex lens, and the optical chopper were all well aligned and positioned with the monochromator in order to maximize the input signal at the monochromator input. For focusing the output signal, a convex lens was positioned at the output of the monochromator. The detector was aligned behind the lens using an xyz-translation stage. The whole setup was enclosed in a measurement chamber, which was flushed with nitrogen $\left(\mathrm{N}_{2}\right)$ gas in order to minimize radiation absorption from carbon dioxide $\left(\mathrm{CO}_{2}\right)$ in the measurement chamber. The frequency of the optical chopper, and the output signal from the detector, were both fed to a lockin amplifier. The monochromator and the lock-in amplifier were connected with a computer for storage of the captured data.

\section{B. Calibration of the Monochromator}

The monochromator setup was initially calibrated in order to characterize the wavelength dependent photosensitivity of the thermopile detector. In the mid-infrared wavelength band, carbon dioxide has a well-defined absorption peak at $4.26 \mu \mathrm{m}$ [24], which could be used as a reference point for the calibration of the monochromator setup. The measurement setup shown in Fig. 6 was used for the calibration process. To achieve a high resolution in the calibration, the width of both entrance and exist slits was reduced to $0.5 \mathrm{~mm}$. The output signal passing 


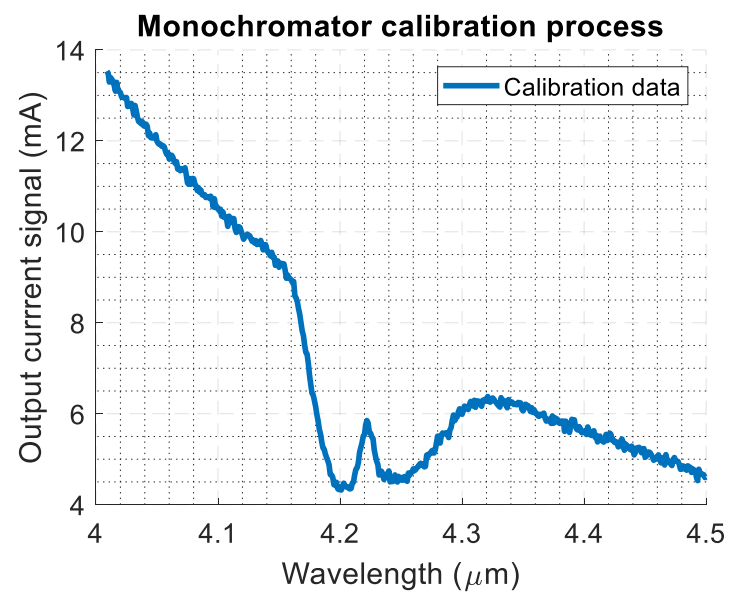

Fig. 7: An IR scan from $4-4.5 \mu \mathrm{m}$ in order to calibrate the monochromator through the carbon dioxide absorption peak.

through the exit slit of the monochromator was focused onto a photodetector using a plano convex lens. The optical chopper frequency was set to $3 \mathrm{~Hz}$, and a scan from $4-4.5 \mu \mathrm{m}$ wavelength with a resolution of $1 \mathrm{~nm}$ was conducted. The output signal from the lock-in amplifier was logged and plotted in Fig. 7. In this figure, we can clearly observe the $\mathrm{CO}_{2}$ absorption peak is at $4.222 \mu \mathrm{m}$, which is shifted about $38 \mathrm{~nm}$ towards the shorter wavelength. The shift in wavelength was noted down and entered into the software to correct the measurements according to calibration value.

\section{MEASUREMENT, RESUlTS AND DisCUSSION}

In this section, the measurement results of the thermopile detector through two different measuring setups will be discussed. First, the thermopile detector was characterization for a voltage response and a photosensitivity using a MEMS based IR source with and without optical filter of $4.26 \mu \mathrm{m}$ wavelength will be present. In the second measurement setup, the photosensitivity and specific detectivity resluts of the thermopile detector for the wavelength range $2.5-5.5 \mu \mathrm{m}$ through a monochromator setup will be discussed and presented.

\section{A. Voltage response measurement for the fabricated detector}

By using bulk values of the materials, the serial resistance of the thermopile detector was estimated to $75 \mathrm{k} \Omega$. However, the serial resistance of the thermopile detector was measured by connecting the device to multi-meter and found to be about $315 \mathrm{k} \Omega$. The resistance of thin films is higher compared to resistance to bulk values reported in [25]. The serial resistance of thermopile detector is 4.2 times higher than the estimated values. Moreover, the contact resistances of the thermocouple junctions also contribute to higher serial resistance compared to theoretical values.

A MEMS based IR-emitter, packaged in a TO39 metal header, without glass window, manufactured by Axetris, was used to measure the voltage responses of both the thermopile detector and a reference detector (HMS J21 thermopile from Heimann Sensor GmbH [26]). The net radiation power of the IR emitter is $55.9 \mathrm{~mW} / \mathrm{m}^{2} \mathrm{~K}^{4}$ at $456 \mathrm{C}$ and gives an IR spectrum range of $2-14 \mu \mathrm{m}$. The IR-emitter is connected to the

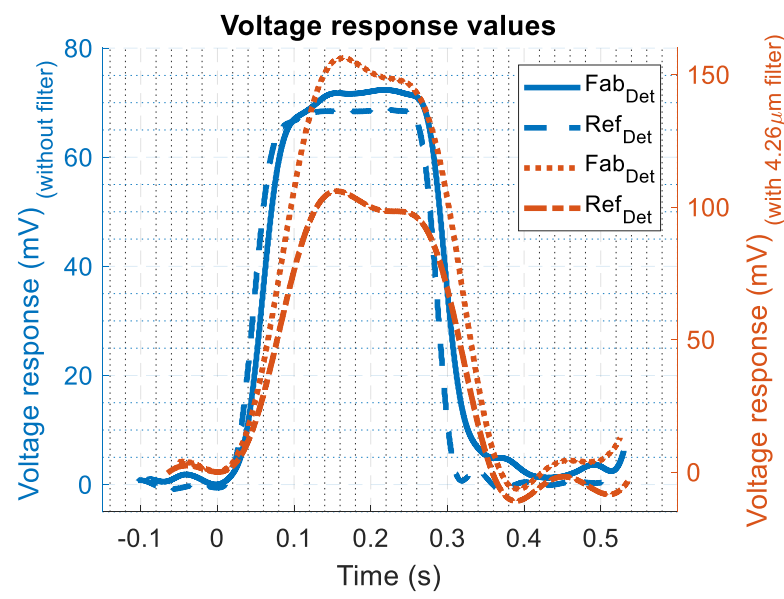

Fig. 8: Both blue lines (solid line and dashed) represents the measurement results for the thermopile and reference detector without optical filter, and both red lines (dotted and dashed) represents the measurement data with an optical filter of wavelength $4.26 \mu \mathrm{m}$.

controller (IRS LabKit G1) which is controlled by a computer software.

For our measurements, the IR-emitter was set at a $50 \%$ duty cycle and $2 \mathrm{~Hz}$ modulation frequency. The measurements were performed by placing both detectors, each at a time, $0.5 \mathrm{~mm}$ away from the IR emitter. The reference detector has a wide absorption band compared to our thermopile detector, which is designed for maximum absorption in a narrow band $3.13-5.5 \mu \mathrm{m}$. This results in a difference in the optical power absorbed in the detector. The voltage responses of both detectors are Fig. 8 (left y-axis). The voltage responses of the thermopile detector and reference detector were measured to $72.4 \mathrm{mV}$ and $68.7 \mathrm{mV}$, respectively. The thermopile detector has a slightly higher voltage response than the reference detector even though it has a narrow band absorber. The radiation flux of the emitter was calculated at $1.76 \mathrm{mWmm}^{-2}$, by using the specific voltage response value, $39 \mathrm{Vmm}^{2} \mathrm{~W}^{-1}$, of the reference detector. For the fabricated thermopile detector, the electric voltage responsivity value was calculated to $41.13 \mathrm{Vmm}^{2} \mathrm{~W}^{-1}$.

Using a pulsed laser diode source of wavelength $1.55 \mu \mathrm{m}$, the time constant of the thermopile detector as well as the reference detector was measured to $21 \mathrm{~ms}$ and $11 \mathrm{~ms}$, respectively. The time constant of the reference detector corresponds well to the specified value given in the datasheet. A previously fabricated SU-8 thermopile detector with a black paint absorber, reported in [27], was measured to have a time constant of $70 \mathrm{~ms}$ using the same laser diode IR source.

\section{B. Voltage response measurement with optical filter}

Carbon dioxide has an absorption peak in the IR spectrum at $4.26 \mu \mathrm{m}$. By introducing an optical band-pass filter at this wavelength, the thermopile detector can be used for the detection of $\mathrm{CO}_{2}$. This system is known as a NDIR system and is commonly used for gas detection.

The second measurement was performed by placing an optical filter with a wavelength of $4.26 \mu \mathrm{m}$ in front of the IR emitter. The voltage responses of the thermopile as well as the reference detector were measured to $156.2 \mathrm{mV}$ and $106 \mathrm{mV}$, respectively. Voltage response values for both detectors were amplified 1000 times by using a low noise amplifier, the 


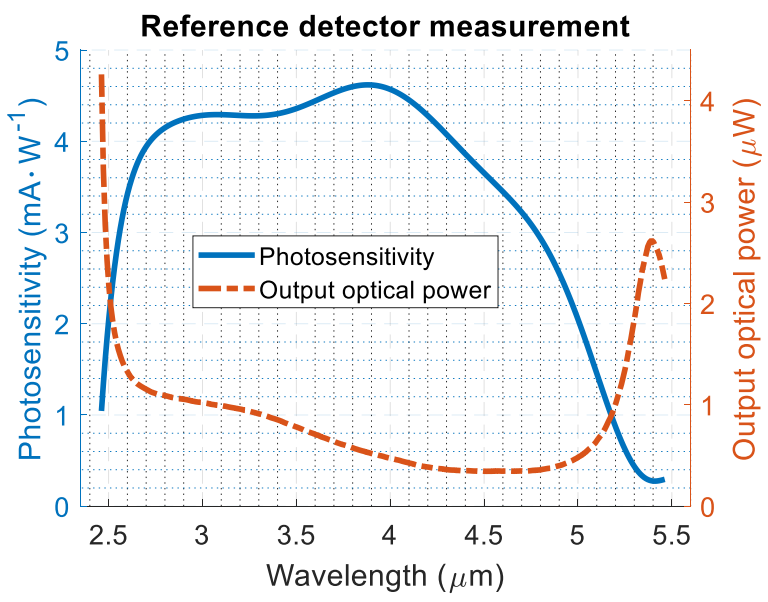

(a)

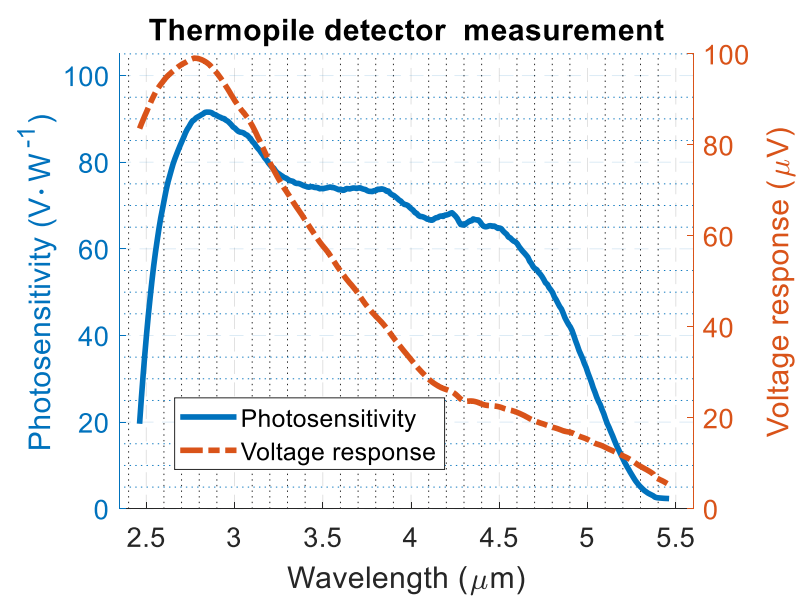

(b)

Fig. 9: (a) A solid line plotted on left y-axis shows photosensitivity results while a dashed dotted line on right y-axis shows IR source optical power in watts for the reference detector, (b) A dashed dotted line on the right y-axis represents voltage response (V) for the thermopile detector, and a solid line plotted on left yaxis shows photosensitivity results of the thermopile detector.

measurement results are Fig. 8 (right y-axis). For the thermopile detector, the photosensitivity value was estimated to $57.5 \mathrm{~V} \cdot \mathrm{mm}^{2} \mathrm{~W}^{-1}$, which is more than $32 \%$ higher than the reference detector. The increased photosensitivity and response speed is a result of high absorption and a reduction in thermal capacitance and conductance due to the integration of an absorber structure.

\section{Characterization of optical output power.}

An InAsSb photo detector from Hamamatsu was used as a reference detector. The reference detector was used to calculate the output optical power of the IR source used in the measurements. The measurement chamber was flushed with $\mathrm{N}_{2}$ about an hour before the measurement was started, and it continued during the measurement to remove contaminations from the surrounding atmosphere in the chamber. The infrared source was supplied with electrical power of $18 \mathrm{~W}$ to get maximum optical power, and the frequency of the optical chopper was set to $3 \mathrm{~Hz}$, and fed back to the lock-in amplifier. The reference detector was well aligned and positioned to get maximum output signal from the exit slit of the monochromator. The entrance and exit slit opening of the monochromator was set at $2 \mathrm{~mm}$. The measurement was performed for the wavelength range of $2.5-5.5 \mu \mathrm{m}$ with a resolution of $10 \mathrm{~nm}$. The photosensitivity value of the reference detector was calculated by using the measured output current signal and the specific detectivity $\left(\mathrm{D}^{*}\right)$ values of the reference detector. Fig. 9a (left y-axis) shows the calculated photosensitivity $\mathrm{mA} \cdot \mathrm{W}^{-1}$ of the reference detector. The optical output power of the emitter was calculated by dividing the output current signal by the photosensitivity value of the reference detector, and the results are plotted in Fig. 9a (right yaxis).

\section{Characterization of the detector spectral response}

The fabricated thermopile detector was mounted on a translation stage, positioned and aligned at the focal point of the plano convex lens to achieve maximum output signal. The measurement setup was flushed with $\mathrm{N}_{2}$ about an hour before the measurement started, and it continued during the measurement to minimize the contaminating effects of the surrounding atmosphere on the results. The entrance and exit slit of the monochromator was set to $2 \mathrm{~mm}$, and the optical chopper frequency was to $3 \mathrm{~Hz}$.

The measurement was taken for a wavelength range of 2.5$5.5 \mu \mathrm{m}$ with the resolution of $10 \mathrm{~nm}$. The voltage response of the thermopile detector is plotted in Fig. 9b (right y-axis), and can be seen to follow the blackbody radiation curve. As presented in Chapter VI section C, the power for the infrared emitter was determined as a function of the wavelength. Hence, the photosensitivity, $\mathrm{V} / \mathrm{W}$, of the thermopile in this wavelength range could also be determined and plotted in Fig. 9b (left yaxis). Here, the maximum photosensitivity value of about $92 \mathrm{~V} / \mathrm{W}$ was found at a wavelength of $2.84 \mu \mathrm{m}$, and decreases gradually with increasing wavelength. At $5 \mu \mathrm{m}$, the photosensitivity was found to be about $30 \mathrm{~V} / \mathrm{W}$. The detector has $65 \mathrm{~V} / \mathrm{W}$ and reference detector has $4.2 \mathrm{~mA} / \mathrm{W}$ photosensitivity at $4.26 \mu \mathrm{m}$ wavelength, respectively. The photosensitivity of the reference detector is in $\mathrm{mA} / \mathrm{W}$ as it is a photo diode detector.

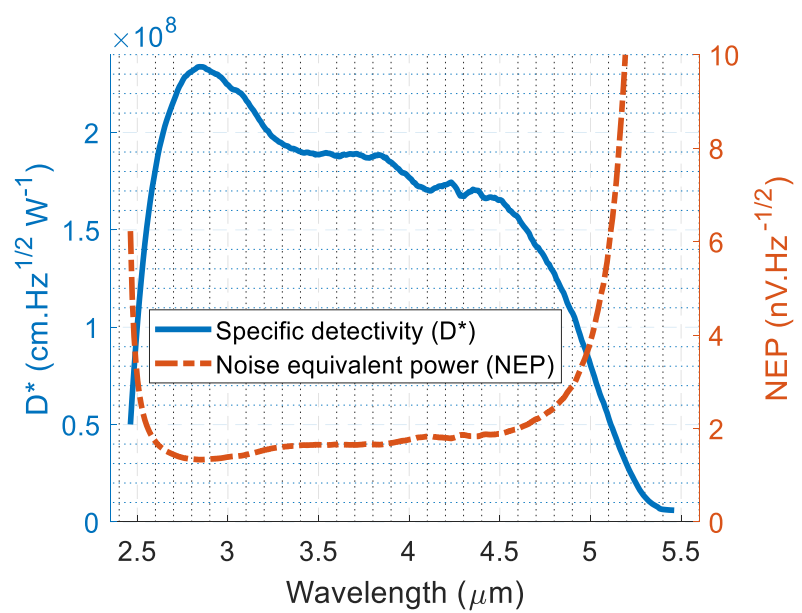

Fig. 10: A solid line plot on the left y-axis shows calculated specific detectivity, and the dashed dotted line plot on the right y-axis represents the noise equivalent power of the thermopile detector. 
The multilayered absorber structure, presented in Chapter III section A, was fabricated and characterized independently. During the integration into the membrane of the thermopile, the layer thickness of the SU-8 epoxy could have some thickness variation due to processing limits. This variation of the SU-8 epoxy could shift the absorber band in wavelength, and thus the multilayered absorption band could differ slightly from the results presented for the separate absorber structure.

The whole purpose of the multilayered structure was to achieve maximum absorption in the mid-infrared region for the use indetection of gases such as $\mathrm{CO}_{2}$ and methane $\left(\mathrm{CH}_{4}\right)$. For example, $\mathrm{CO}_{2}$ and $\mathrm{CH}_{4}$ have strong absorption peaks at $4.26 \mu \mathrm{m}$, and at $3.3 \mu \mathrm{m}$, respectively. For these wavelengths, the detector has shown a photosensitivity values of about $65 \mathrm{~V} \cdot \mathrm{W}^{-1}$ and $80 \mathrm{~V} \cdot \mathrm{W}^{-1}$, as shown in Fig. $9 \mathrm{~b}$ (left y-axis).

The characterization verify that the fabricated thermopile with an intregrated multilayered absorber has high photosensitivity in a broad wavelength range. The specific detectivity $\left(\mathrm{D}^{*}\right) \mathrm{cm} \cdot \mathrm{Hz}^{1 / 2} \cdot \mathrm{W}^{-1}$ and noise equivalent power (NEP) $\mathrm{nV} \cdot \mathrm{Hz}^{-1 / 2}$ was calculated from the measurement data, and plotted in Fig. 10. Here, the thermopile detector shows a specific detectivity value that varies from $8.0 \times 10^{7}$ to $2.4 \times 10^{8}$ $\mathrm{cm} \cdot \mathrm{Hz}^{1 / 2} \cdot \mathrm{W}^{-1}$ for a wavelength range of $2.84-5 \mu \mathrm{m}$. The detector has dectivity of $1.9 \times 10^{8}$ and referecne detector has $9 \times 10^{8}$ value, which is compartivily lower then reference detctor because reference detecotor is silicon based photo detector.

With the current design parameters of the multilayered absorber, the thermopile could be used in detection systems for a mid infrared region of about $3-5 \mu \mathrm{m}$. However, by tuning the absorber structure parameter, it could be used for other adjacent wavelength regions.

\section{SUMMARY AND CONCLUSION}

In this paper, we have presented an integration process of a mid-infrared absorber (multilayered $\lambda / 4$ ) structure into the SU8 membrane of a thermopile detector. Measurement results of the thermopile detector have been presented. The absorber structure shows an absorption of more than $95 \%$ in the range of 3.13-5.47 $\mu \mathrm{m}$. Using standard fabrication techniques, a complete fabrication process has been demonstrated. The serial resistance of the detector was measured to about $315 \mathrm{k} \Omega$. A MEMS based infrared emitter was used for initial e characterization of voltage response. These measurements were taken in two stages with and without an optical filter: in the first stage, the voltage response of the thermopile detector was measured using the entire radiation band of the emitter. The voltage responses were measured to $72.4 \mathrm{mV}$ and $68.7 \mathrm{mV}$ for the thermopile and reference detector, respectively. The optical radiation power of the emitter was estimated to $1.76 \mathrm{mWmm}^{-2}$ by using the specified voltage response of the reference detector. For the presented thermopile detector, the photosensitivity was calculated to $41.13 \mathrm{Vmm}^{2} \mathrm{~W}^{-1}$. The timeconstant value of the thermopile detector was estimated to around $21 \mathrm{~ms}$. This is a noticeable reduction compared to values presented previously, caused by integrating the absorber structure and, therefore, reducing the thermal capacitance. In the second stage, an optical bandpass filter with a narrow passband at a wavelength of $4.26 \mu \mathrm{m}$ was placed between emitter and detector. With this setup, the voltage response was measured to $156.2 \mathrm{mV}$ and $106 \mathrm{mV}$ for the presented detector and the reference detector, respectively. The photosensitivity of the detector presented was increased to $57.5 \mathrm{Vmm}^{2} \mathrm{~W}^{-1}$. This increase in photosensitivity was caused by an efficient absorber. The integration of the absorber in a membrane material consisting of a material with low thermal conductivity contributes the increasing the photosensitivity.

The spectral response of the detector was characterized for the wavelength range of $2.5-5.5 \mu \mathrm{m}$. The characterization was performed by using a mononchromator setup. The output signal from the monochromator was modulated using an optical chopper and focused on the detector. For the noise reduction and amplification, the chopper frequiency and the output signal from the detector were fed to a lock-in amplifier. The optical input power was characterized by using a referece photodetector with the specified photosensitivity. The detector has shown a maximum photosensitivity value of $92 \mathrm{~V} / \mathrm{W}$ at a wavelength of $2.84 \mu \mathrm{m}$. This value gradually decreased with an increase in wavelength to $30 \mathrm{~V} / \mathrm{W}$ at about $5 \mu \mathrm{m}$. For the region

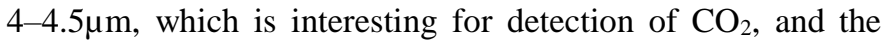
region 3.1-3.5 $\mu \mathrm{m}$ that is interesting for the detection of $\mathrm{CH}_{4}$, the detector has shown a photosensitivity value higher than $65 \mathrm{~V} / \mathrm{W}$. The thermopile detector has a photosensitivity ranging from 30 to $92 \mathrm{~V} \cdot \mathrm{W}^{-1}$ and a specific detectivity value from $8.0 \times 10^{7}$ to $2.4 \times 10^{8}$ for the wavelength range of $2.84-5 \mu \mathrm{m}$. The detector presented was mainly designed for the detection of gases in the mid-infrared region, such as $\mathrm{CO}_{2}$ and $\mathrm{CH}_{4}$. However, for the detection of other gases, tuning the absorption parameters could shift the absorption band up or down in the wavelength spectrum.

\section{ACKNOWLEDGMENT}

The research has been carried out in the STC (Sensible Things that Communicate) research centre at Mid Sweden University. We would like to acknowlege SensAir AB

\section{REFERENCES}

[1] A. Rogalski, "Infrared detectors: an overview," Infrared Phys. Technol., vol. 43, no. 3-5, pp. 187-210, Jun. 2002.

[2] J.-L. Lai and G.-D. J. Su, "Microbolometer SU-8 photoresist microstructure with cytochrome $\mathrm{c}$ protein as a sensing pixel for microbolometer," in SPIE Optical Engineering + Applications, 2012, p. 85120V.

[3] "SU-8 2000 Datasheet [Online]. Available: www.microchem.com/ products/su_eight.htm."

[4] A. Levin, "A numerical simulation tool for infrared thermopile detectors," Int. Conf. Thermoelectr. ICT, Proc., vol. 2005, pp. 464467, 2005.

[5] W. Lang, "Heat transport from a chip," IEEE Trans. Electron Devices, vol. 37, no. 4, pp. 958-963, 1990.

[6] C. G. Mattsson, G. Thungstrom, K. Bertilsson, H.-E. Nilsson, and H. Martin, "Design of a Micromachined Thermopile Infrared Sensor With a Self-Supported SiO2/SU-8 Membrane," IEEE Sens. J., vol. 8, no. 12, pp. 2044-2052, 2008.

[7] I. Mellouki, N. Bennaji, and N. Yacoubi, "IR characterization of graphite black-coating for cryogenic detectors," Infrared Phys. Technol., vol. 50, pp. 58-62, 2007.

[8] X. Chenyang, W. Kaiqun, T. Liang, Z. Wendong, and X. Jijun, "IR characterization of carbon-black for thermopile infrared detectors," in 2010 IEEE 5th International Conference on Nano/Micro Engineered and Molecular Systems, 2010, pp. 963-966.

[9] S. Bauer, S. Bauer-Gogonea, W. Becker, R. Fettig, B. Ploss, W. Ruppel, and W. von Münch, "Thin metal films as absorbers for 
infrared sensors," Sensors Actuators A Phys., vol. 37-38, pp. 497501, Jun. 1993. [10] K. C. Liddiard, "Application of interferometric enhancement to selfpp. 379-387, 1993.

[11] M. Ahn, Y. H. Han, and S. Moon, "A novel infrared absorbing structure for uncooled infrared detector," Curr. Appl. Phys., vol. 7, pp. 617-621, 2007.

[12] S. Ashraf, C. G. Mattsson, G. Thungström, and H. Rödjegård, "Integration of an interferometric IR absorber into an epoxy membrane based CO 2 detector," J. Instrum., vol. 9, no. 05, pp. C05035-C05035, May 2014.

[13] P. Eriksson, J. Y. Andersson, and G. Stemme, "Interferometric, low thermal mass IR-absorber for thermal infrared detectors," Phys. Scr., vol. T54, no. T54, pp. 165-168, Jan. 2007.

[14] S. Ashraf, C. G. Mattsson, G. Thungstrom, and H. Rodjegard, "Design of a multilayered absorber structure based on SU-8 epoxy for broad and efficient absorption in Mid-IR sensitive thermal detectors," in IEEE SENSORS Proceedings, 2014.

[15] S. Ashraf, C. G. Mattsson, and G. Thungström, "Fabrication of a mid-IR sensitive thermopile detector," in IEEE SENSORS Proceedings, 2016.

[16] Z. Djuric, "New generation of thermal infrared detectors," Proc. Int. Conf. Microelectron., vol. 2, pp. 559-64, 1995.

[17] A. Rogalski, "Infrared detectors: Status and trends," Prog. Quantum Electron., vol. 27, pp. 59-210, 2003.

[18] E. D. Palik, Handbook of Optical Constants of Solids. Elsevier, 1997.

[19] S. Ashraf, C. G. Mattsson, M. Fondell, A. Lindblad, and G. Thungström, "Surface modification of SU-8 for metal/SU-8 adhesion using RF plasma treatment for application in thermopile detectors," Mater. Res. Express, vol. 2, no. 8, p. 086501, Aug. 2015.

[20] T. Takahashi, M. Makihata, M. Esashi, and S. Tanaka, "Evaluation and Application of Resist for Alkaline Wet Etching," IEEJ Trans. Sensors Micromachines, vol. 130, no. c, pp. 421-425, 2010.

[21] TRIAX 180 Monocrhomator,

"http://www.horiba.com/scientific/products/opticalspectroscopy/spe ctrometers-monochromators/triax/triax-180-202/."

[22] Grating,

"http://www.horiba.com/fileadmin/uploads/Scientific/Documents/G ratings/grating-curve-510-23.pdf.".

[23] Scitec Instruments Ltd,

"http://www.scitec.uk.com/irsources/pdf/ir18-ir19.pdf.".

[24] HITRAN,

"http://vpl.astro.washington.edu/spectra/co2hitran2004imagesmicro ns.htm.".

[25] K. M. Leung, "Electrical resistivity of metallic thin films with rough surfaces," Phys. Rev. B, vol. 30, no. 2, pp. 647-658, Jul. 1984. "HMS J21 Datasheet, Rev.07 / 01.10.2004 [Online.] Availabe: http://www.boselec.com/products/detir.html."

27] C. G. Mattsson, G. Thungström, K. Bertilsson, H.-E. Nilsson, and H. Martin, "Fabrication and characterization of a design optimized SU-8 thermopile with enhanced sensitivity," Meas. Sci. Technol., vol. 20, p. 115202, 2009.

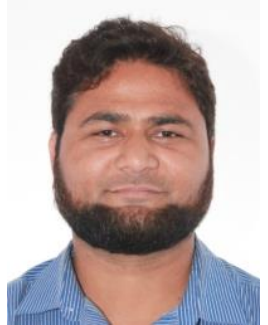

Shakeel Ashraf was born in the small town of Lalamusa in Pakistan in 1984. He received his Master's degree in Physics from the University of Punjab in 2008, and a Master's degree in Electrical Engineering from Mid Sweden University, Sundsvall Sweden, in 2011. He is currently working towards a $\mathrm{PhD}$ degree in infrared sensors, in the Sensors Group, the Department of Electronics Design, Mid Sweden University. His research mainly focuses on semiconductor process technologies related to infrared detectors, such as bolometers and thermopiles.

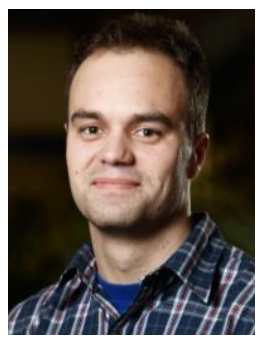

Claes G. Mattsson was born in Söderhamn, Sweden, in 1978. He received his M.Sc. degree in Electrical Engineering from Mid Sweden University, Sundsvall Sweden, in 2002. In 2009, he presented his $\mathrm{PhD}$ thesis, "Design, fabrication and optimization of thermal radiation detectors based on thin polymer membranes" at Mid Sweden University. Today, he works as Head of the Department of Electronics Design at Mid Sweden University. His research mainly focuses on semiconductor process technologies related to radiation detectors and infrared detectors, such as bolometers and thermopiles.

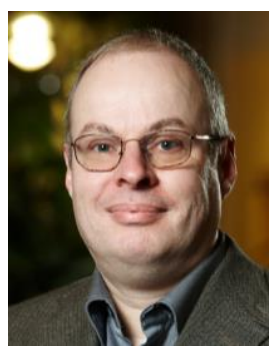

Göran Thungström was born in Härnösand, Sweden, in 1960. He received his $\mathrm{PhD}$ degree in Solid-state Electronics from the Royal Institute of Technology, Stockholm, Sweden, in 2000. In 2000, he accepted a position as a University Lecturer at Mid Sweden University. One of his initial tasks was to build up and develop the processing of semiconductors in a newly built cleanroom. A second task involved starting up a research group in sensors and to employ $\mathrm{PhD}$ students for this group. Currently, the focus of the research is on radiation detectors, mainly in silicon technology and in related micromechanical technology for developing infrared detectors. 